\title{
NiCARAGUA 2008: POLARIZACIÓN Y PACTOS
}

\author{
Nicaragua 2008: Pacts and Polarization
}

\section{SALVADOR MARTÍ I PUIG}

Universidad de Salamanca-España

\section{RESUMEN}

El año 2008, el segundo de la "nueva" administración de Daniel Ortega, estuvo marcado por la polarización política, la constatación de que El Pacto entre Ortega y Alemán se mantenía a pesar de las declaraciones de los miembros del partido liberal y de la dificultad de que las políticas sociales implementadas por el gobierno se tradujeran en una mejora del nivel de vida de los ciudadanos más pobres. En dicho marco, Daniel Ortega mantuvo una notable continuidad con el año anterior tanto respecto a la política exterior como a la doméstica, destacando el incremento del poder de la Primera Dama que se convirtió en Ministro de la Presidencia. Finalmente cabe destacar la trascendencia que tuvieron las elecciones municipales celebradas el 9 de marzo.

Palabras clave: Nicaragua, sandinismo, FSLN, Daniel Ortega, Rosario Murillo, pactos, ALBA, elecciones municipales.

\begin{abstract}
2008, which was the second year of Daniel Ortega's "new administration" was marked by political polarisation, the confirmation that the Pact between Ortega and Alemán was still in place, despite declarations made by members of the Liberal Party, and the difficulty in translating the social policies implemented by the government into an improvement in the quality of life for the poorest citizens. In this context, Daniel Ortega maintained a notable continuity with the previous year with regard to both foreign and domestic policy, particularly the increase in the power of the First Lady, who became Minister of the Presidency. Finally, it is necessary to highlight the importance of the municipal elections held on the 9th of November.
\end{abstract}

Key words: Nicaragua, sandinismo, FSLN, Daniel Ortega, Rosario Murillo, pacts, ALBA, local elections. 


\section{LEGADOS, UNA CONSTATACIÓN Y RETOS}

El año 2008, el segundo de la "nueva" administración de Daniel Ortega, inició con un pésimo legado, una constatación y diversos retos. ${ }^{1}$ El pésimo legado fue el enfrentamiento institucional y la creciente polarización que produjo el primer año de la administración Ortega. Esta administración se caracterizó por su poca voluntad de consenso y su discrecionalidad a la hora de llevar a cabo temas críticos. Fruto de esta actitud en determinados períodos la oposición parlamentaria se aglutinó en el llamado "Bloque contra la dictadura". El caso más llamativo fue el conflicto que hubo entre Ejecutivo y Legislativo a raíz de la legalización los Consejos del Poder Ciudadano (CPC) como espacios de participación y distribución de recursos -articulados dentro del Consejo Nacional de Planificación Económica y Social (CONPES)- el día 29 de noviembre de 2007 a través de dos decretos presidenciales. Ante estos decretos los 52 diputados de la oposición aprobaron una ley (la L630) que transformaba la naturaleza de los CPC. Sin embargo la Ley 630 terminó siendo declarada inconstitucional por la Corte Suprema de Justicia (controlada por el FSLN), generando una viva protesta de los diputados opositores quienes decidieron no aprobar el Presupuesto de 2008 el 16 de diciembre de 2007 como muestra de su rechazo ${ }^{2}$. Frente a esta posición de fuerza contra el FSLN, Daniel Ortega se refirió a ellos con calificativos poco edificantes, como los de "reptiles", "somocistas", "financiados por el imperio" o de "perros rabiosos". Así las cosas, como se ha señalado, el segundo año del gobierno Ortega empezó con una herencia envenenada: la polarización y el enfrentamiento. Sin embargo, ambas incrementarían durante el 2008, sobre todo a raíz de las elecciones municipales de noviembre de 2008.

La constatación (que en un inicio era un interrogante) fue la solidez de la alianza entre Alemán y Ortega establecida con la firma de El Pacto en el año 2000. De la continuidad de este acuerdo (que politizó y partidarizó la administración pública, el Poder Judicial y los órganos autónomos del Estado), dependía que Daniel Ortega pudiera aprobar su legislación en la Asamblea Nacional y que Arnoldo Alemán pudiera soñar con su excarcelación y, por lo tanto, su vuelta a la arena política. ${ }^{3}$

Así, uno de los temas más relevantes del período analizado fue el status de Arnoldo Alemán. En este sentido el año empezó con la autorización del Poder Judicial, dictada el 9 de enero, para que el líder del PLC pudiera participar en una importante reunión de los liberales para enfrentar conjuntamente al FSLN en los comicios municipales de noviembre y suscribir una especie de "acuerdo de unidad". ${ }^{4}$ Además un sector del liberalismo propuso a la Asamblea

1 Para una comprensión del presente artículo se recomienda la lectura del texto sobre Nicaragua que aparece en el Anuario de 2007 de la misma revista y que analiza los primeros pasos de la administración Ortega y el contenido de El Pacto (Martí 2008b).

2 Posteriormente, el día 11 de enero de 2008 se aprobó el Presupuesto por el voto a favor de diversos diputados liberales allegados al ex presidente y reo Arnoldo Alemán a cambio de una decisión judicial (dictada en enero de 2008) que dio al líder del PLC la posibilidad de moverse libremente por el país.

3 Tal como ocurrió a inicios del año siguiente, en enero de 2009, cuando Arnoldo Alemán fue absuelto por la justicia nicaragüense. El análisis de este evento, sin embargo, pertenece al Anuario del año siguiente.

4 Debe señalarse que los liberales (que son la oposición mayoritaria al FSLN) se enfrentaron divididos en dos formaciones (el PLC y ALN) a Ortega. Gracias a esta división Daniel Ortega ganó la Presidencia de la República. Para mayor información de las elecciones nicaragüenses de 2006 véase: (Martí 2008a). 
Nacional, el día 7 de febrero, la posibilidad de redactar una Ley de Amnistía para que Arnoldo Alemán y todos los encausados por corrupción bajo su administración pudieran salir libres y sin cargos. La aceptación de esta demanda por parte del FSLN supuso la ruptura del llamado "Bloque contra la dictadura" en la Asamblea Nacional y la aprobación del Presupuesto de la Nación el día 12 de febrero. A raíz de dicho evento a inicios de año se constató la permanencia del pacto Ortega-Alemán debido a las atenciones que el Poder Judicial tuvo a lo largo del año con el ex Presidente y a la capacidad del gobierno para poder romper la cohesión de la oposición obteniendo el voto a favor de los 8 a 10 diputados liberales leales a Alemán y conseguir así mayorías suficientes para aprobar sus iniciativas.

Esta constatación -la permanencia de El Pacto- despertó recelos y críticas de múltiples sectores de la sociedad civil, destacando el rol de la Conferencia Episcopal, de las organizaciones no gubernamentales (ONG) y de la prensa, que denunciaron el "secuestro" de las instituciones por parte de los dos caudillos políticos del país. Frente a esta actitud opositora, el gobierno de Ortega desarrolló estrategias de disuasión e intimidación. Entre ellas destacaron, por un lado, la denuncia que hizo un juez a la directiva del periódico La Prensa por delito de injurias contra el gobierno en abril y, por otro, la acusación a un centro de investigación ${ }^{5}$ y a dos organizaciones no gubernamentales ${ }^{6}$ de blanquear dinero y de actuar contra el gobierno en octubre de 2008. ${ }^{7}$ Esta estrategia de la administración -que reproducía una política ya adoptada años atrás por el anterior mandatario Arnoldo Alemán (Kampwirth 2004)- tenía como objetivo acallar las voces más críticas de la sociedad civil, si bien el efecto de esta intervención fue la contraria: una condena de la comunidad internacional y de la opinión pública doméstica.

Finalmente, en cuanto a los múltiples retos de la administración se concentraron en el ámbito de las políticas sociales y de lucha contra la pobreza, pues el mensaje de la administración Ortega se focalizó en esta temática a pesar de que en 2007 el poder adquisitivo de los salarios ${ }^{8}$ cayó un 13\% y el crecimiento del PIB fue menor que el año anterior (en 2008 fue de un $3,5 \%$ frente el $4,2 \%$ de 2007 ).

En este contexto las dos primeras medidas del año fueron el acuerdo entre el gobierno y los sindicatos para aumentar un 15\% el salario mínimo durante el mes de enero y el estrechamiento de la relación de Nicaragua con los países de la Alternativa Bolivariana de las Américas (ALBA), sobre todo con Venezuela, ${ }^{9}$ país al que, según Ortega, había

5 El centro en cuestión era el Centro de Investigaciones de la Comunicación (CINCO).

6 Una era una ONG extranjera, Oxfam-UK, y otra una nicaragüense llamada Movimiento Autónomo de Mujeres, MAM.

7 El embargo con orden judicial de los archivos (físicos e informáticos) a las Oficinas de CINCO y MAM generaron mucha tensión, así como las declaraciones de los Viceministros de Relaciones Exteriores refiriéndose a las organizaciones como protagonistas de una estrategia política de confrontación ideológica; con todo, la acusación legal fue la de "triangulación" de recursos provenientes de la cooperación internacional, vinculando incluso las organizaciones citadas al narcotráfico.

8 Cabe señalar que sólo el 25\% de la población económicamente activa de Nicaragua tiene un contrato salarial formalizado.

9 Es importante anotar que el país donde más importa Nicaragua continúa siendo los Estados Unidos (con un $24,5 \%$ del total de las compras nicaragüenses), seguido de El Salvador y Honduras. Sin embargo, en estos últimos dos años Venezuela ha pasado de tener un rol irrelevante a significar un 19\% de éstas. Es posible que 
salvado a Nicaragua de un "colapso económico". Cabe señalar, sin embargo, que el FMI -en su visita de dos semanas a inicios de febrero- criticó tanto las medidas del gobierno respecto al alza de salarios como la ausencia de transparencia de los fondos que llegan de la administración venezolana. ${ }^{10}$

Enfrentar la situación socioeconómica del país fue el reto más exigente del gobierno, ya que Nicaragua es un país donde el $77.8 \%$ de la población vive con menos de dos dólares diarios y el $42.6 \%$ con menos de uno. Y en el que, según expuso la encuestadora M\&R en el mes de mayo, el $66.3 \%$ de la gente quiere irse del país: la mitad por cuestiones políticas y la otra mitad por falta de empleo.

Así las cosas, el objetivo del presente texto es, en primer lugar, intentar analizar cómo, desde el legado de polarización al que se hace referencia, desde la continuidad de El Pacto firmado por Ortega y Alemán, y con el reto de luchar contra la pobreza, los políticos nicaragüenses desarrollaron sus tareas legislativas y gubernamentales. En segundo lugar exponer e interpretar lo acontecido en elecciones municipales de noviembre y, finalmente, hacer un balance sobre el funcionamiento de la democracia en el país.

\section{DINÁMICA INSTITUCIONAL}

\section{Legislar en un gobierno dividido}

Al igual que el año anterior la permanencia de El Pacto supuso que durante toda la legislatura se mantuviera en la Asamblea Nacional una dinámica partidaria con coaliciones sin concordancia ideológica. El hecho de que el FSLN sólo contara con 38 de los 90 escaños supuso que la aprobación de las leyes se hiciera con la obtención de votos de diputados pertenecientes al PLC, generalmente los más fieles a Alemán. Sin embargo, se vio también como algunos miembros del PLC se separaban de la línea que postulaba Alemán y se acercaban a posiciones próximas a la ALN. Esta fluidez en las lealtades de los diputados opositores generó una gran plasticidad y tacticismo a la hora de aprobar leyes en la Asamblea Nacional. Al final del año el balance final de leyes aprobadas no fue especialmente negativo: el gobierno consiguió que se aprobaran 31 leyes de las 73 propuestas presentadas a lo largo de las 46 sesiones celebradas, ${ }^{11}$ así la tasa de éxito fue de un $42 \%$, un $10 \%$ de éxito más que el año anterior. En la figura que sigue se expone la actividad legislativa del año.

se mantenga esta tendencia debido a la firma -en la VI Cumbre del ALBA celebrada a finales de enero en Caracas- de un "acuerdo de seguridad alimentaria" entre los dos países.

10 Se calcula que los fondos que Nicaragua ha recibido de la asociación con Venezuela a través de la empresa ALBA-CARUNA han sido cuantiosos. Del "Fondo ALBA" se calcula la obtención de 71.884.000 dólares, que se han destinado a los programas sociales de "calles para el pueblo", de construcción de viviendas sociales, "usura cero" y "hambre cero". También se ha señalado la apertura de una línea de crédito del Banco del Alba con el Banco Nacional de Desarrollo (BANDES) que ha aportado 36.958.600 dólares. Algunas fuentes han apuntado que el total de la ayuda venezolana fue de 520.516 .500 dólares durante el año 2008. Pero también cabe anotar que frente a la caída del precio del crudo Venezuela declaró a mediados de 2008 que no construiría la refinería que prometió el año anterior en el municipio de Nagarote, en Nicaragua, cuya inversión era de 4.000 millones de dólares. Para mayor información véase: http:/ / www.envio.org.ni/articulo/3752.

11 En 2007 hubo un total de 64 sesiones ordinarias realizadas. Un total de 18 sesiones menos en 2008 con relación a 2007. 
Labor legislativa Nicaragua 2007-2008

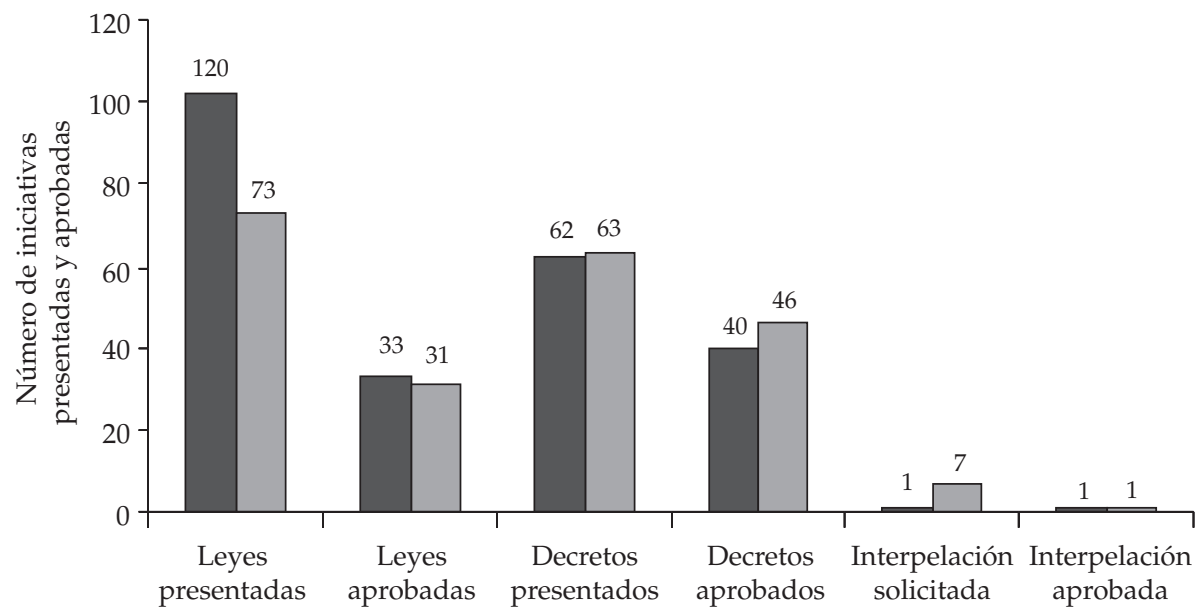

Tipo de normas

$2007 \square 2008$

Fuente: Elaboración propia.

De entre las leyes aprobadas destacaron, por su trascendencia económica y social y por las tensiones políticas provocadas, las doce leyes que se presentan en el siguiente cuadro. Sin embargo, las leyes cuya aprobación provocaron mayor tensión durante la legislatura de 2008 fueron las del presupuesto, la de la de extensión de licencia del espectro radioeléctrico $\mathrm{y}$, sobre todo, la que regulaba los aspectos de las inminentes elecciones municipales. Por otro lado cabe señalar que finalmente, en 2008, se anuló la Ley Marco, que fue diseñada por Ortega y Alemán en 2005 para recortar los poderes del entonces Presidente de la República, Enrique Bolaños. De dicha ley sólo se mantuvo (con el voto a favor de 80 diputados) la capacidad de que la Asamblea Nacional pudiera vetar el nombramiento de Ministros y Viceministros y de nombrar embajadores.

Además de la legislación aprobada cabe destacar también dos comparecencias en la Asamblea Nacional que generaron una notable polémica. La primera fue la del mismo Presidente de la República para explicar el procedimiento de selección del listado propuesto para la elección de magistrados de la Corte Suprema de Justicia, hecho que generó el rechazo unánime de la oposición que presionó con fuerza para que las ternas de los candidatos fueran consultadas a organizaciones de sociedad civil para poder atenuar la politización del Poder Judicial. La segunda comparecencia fue la que realizó el Ministro de Hacienda para explicar los acuerdos y procedimientos para la gestión, ejecución y administración de los recursos provenientes del ALBA, así como la adjudicación de concesiones económicas que realizó el gobierno con diputados de la oposición para la aprobación de presupuesto de 2008. En ambas sesiones el Presidente del Legislativo tuvo que expulsar del hemiciclo a diputados para poner orden en la sala. 
Leyes más significativas de 2008

\begin{tabular}{|c|c|c|}
\hline En el ámbito económico & En el ámbito social & En el ámbito civil \\
\hline $\begin{array}{l}\text { Ley de fomento a la pequeña y mediana } \\
\text { empresa }\end{array}$ & $\begin{array}{l}\text { Ley de igualdad y derechos } \\
\text { de oportunidades }{ }^{12}\end{array}$ & $\begin{array}{l}\text { Reforma a Ley de } \\
\text { Amparo }\end{array}$ \\
\hline $\begin{array}{l}\text { Ley de Presupuesto general de la } \\
\text { República } 2008\end{array}$ & $\begin{array}{l}\text { Ley de Adición a la Ley } \\
\mathrm{N}^{\mathrm{o}} 461 \text {, Ley que Autoriza } \\
\text { al Poder Ejecutivo la } \\
\text { Cancelación de Adeudos } \\
\text { y Compra de Venta de } \\
\text { Viviendas de Interés } \\
\text { Social }^{13}\end{array}$ & $\begin{array}{l}\text { Ley de transparencia } \\
\text { para las entidades y } \\
\text { empresas del Estado } \\
\text { de Nicaragua }\end{array}$ \\
\hline $\begin{array}{l}\text { Ley del Sistema de Sociedades de } \\
\text { Garantías Recíprocas para las Micro, } \\
\text { Pequeña y Mediana Empresa }\end{array}$ & $\begin{array}{l}\text { Ley para distribución y } \\
\text { uso responsable de energía } \\
\text { eléctrica }^{14}\end{array}$ & $\begin{array}{l}\text { Ley de protección a } \\
\text { personas refugiadas }\end{array}$ \\
\hline \multicolumn{3}{|l|}{$\begin{array}{l}\text { Ley de Modificación a la Ley Anual de } \\
\text { Presupuesto General de la República } \\
2008 .\end{array}$} \\
\hline \multicolumn{3}{|l|}{$\begin{array}{l}\text { Ley de Reforma y Adiciones a la } \\
\text { Ley N }{ }^{\circ} 601 \text {, "Ley de Pomoción de la } \\
\text { Competencia"15. }\end{array}$} \\
\hline $\begin{array}{l}\text { Ley de Prórroga a las Licencias de } \\
\text { las Empresas, Personas Naturales o } \\
\text { Jurídicas que Operan Radio, Televisión } \\
\text { y Tele Cable }\end{array}$ & & \\
\hline
\end{tabular}

Fuente: Elaboración propia.

Finalmente, hubo un debate que se mantuvo en 2008 -heredado del año anterior- pero que no se concretó ni se llevó a votación en la Asamblea Nacional. El debate era el de reforma de la Constitución para convertir el sistema político nicaragüense en un régimen parlamentario. Dicho tema ya fue puesto en diciembre de 2007, pero cuando el FSLN

12 Ley con la que la bancada de gobierno y el gobierno mismo trató de reivindicarse con la opinión pública y organismos (nacionales e internacionales) que le han cuestionado la penalización del aborto terapéutico.

13 Ley presentada como la base para la reactivación del sector de la construcción y especialmente para la reducción del déficit habitacional. Fue consensuada con la Cámara de la Construcción y de la Vivienda (Empresa privada) y con activistas de los CPC y del Movimiento Comunal.

14 Esta ley no fue sancionada por el Presidente de la República, sin embargo el presidente de la Asamblea Nacional la publicó y la puso en vigencia.

15 Ley que define el funcionamiento del Tribunal "precompetencia", en el que la empresa privada ha puesto especial interés por los temas de monopolio y competencia desleal, tanto en el caso de empresas privadas como del Estado que ofrecen servicios.

16 Con esta ley se extiende la concesión para el uso del espectro radioeléctrico, especialmente de los canales de televisión. En este contexto la mayoría de los programas matutinos de debate redujeron los enfoques críticos al gobierno para asegurar su continuidad. 
constató que no lograría los votos necesarios para su aprobación lo pospuso. ${ }^{17}$ Sin embargo, durante el mes de octubre de 2008 el jefe del grupo parlamentario del FSLN, Edwin Castro, reabrió el debate con la presentación de un libro titulado Parlamentarización del sistema político nicaragüense realizada en la Corte Suprema de Justicia, en la que participaron magistrados (de sensibilidad liberal y sandinista) de dicha Corte y del Consejo Supremo Electoral. En este debate se constató que los sandinistas y algunos miembros del PLC estarían dispuestos a avanzar en la "parlamentarización" del régimen político, pero la mayoría de la clase política -incluso Arnoldo Alemán-advirtieron que uno de los riesgos de dicho modelo era la reelección indefinida. Finalmente, una vez más, el FSLN no lo propuso en la Asamblea Nacional, al constatar la falta de votos para que prosperara una primera votación a favor de la reforma, ${ }^{18}$ y el tema desapareció de la agenda.

\section{La administración Ortega: Entre la patrimonialización y el activismo focalizado}

Respecto al gobierno liderado por Ortega en el segundo año de su administración, hubo muy pocos cambios, tanto en el diseño e implementación de políticas como en los responsables de los Ministerios, cuyos miembros mantuvieron su bajo perfil. A nivel personal el único cambio relevante en el gabinete fue la formalización del rol que ya venía ejerciendo la Primera Dama, Rosario Murillo, en el Ejecutivo al ser nombrada a inicios de 2008 Coordinadora del Consejo de Comunicación y Ciudadanía, convirtiéndose así en el miembro del gabinete con mayor poder después (¿o a la par?) del Presidente de la República. ${ }^{19}$ Este elemento, junto con la presencia de hijos y nietos de la familia "OrtegaMurillo" en diversos cargos estatales y funciones públicas (así como su presencia en viajes diplomáticos), introdujo en el debate político nacional el tema de la patrimonialización del gobierno y del nepotismo. Un tema nada baladí si se tiene en cuenta que en la historia nicaragüense la presencia de linajes en la vida política ha sido recurrente. Pero además del tema de familiar, el esquema de concentración del poder en la pareja presidencial supuso una mayor centralización de las actividades del gobierno, tanto en el diseño de las políticas -que siguieron dependiendo más de los asesores presidenciales que de los ministros- como en la implementación, sobre todo el de las políticas sociales financiadas con el dinero que fluye de la cooperación venezolana y que muchas veces se distribuye a través de los CPC.

Respecto a las políticas públicas implementadas durante el año 2008, éstas mantuvieron la misma contradicción del año pasado. ${ }^{20}$ Por un lado, se constató una total continuidad

17 En este caso eran 56 votos para aprobar la reforma, ya que se requiere una mayoría cualificada para enmendar la Ley Fundamental.

18 La Constitución exige que las reformas constitucionales deben ser votadas en dos años legislativos consecutivos para que surjan efecto.

19 Cabe señalar también que la Primera Dama ya ejercía un importante poder formal en el FSLN y en la implementación de políticas públicas al ser la Presidenta de los CPC.

20 Según Rose Spalding (2009) es cierto que la administración Ortega planteó, por primera vez en 16 años, políticas sociales activas contra la pobreza, pero para atajar la pobreza no basta dichas políticas, que son sectoriales y muy limitadas, sino que es preciso el abandono de medidas económicas ortodoxas. 
respecto a las anteriores políticas neoliberales ${ }^{21}$ y, por otro, se desplegó -con más propaganda que eficacia- una batería de políticas sociales focalizadas para erradicar la pobreza a través de los programas Hambre Cero, Usura Cero, Calles para el Pueblo y Desempleo Cero.

También cabe anotar que muchas de las políticas sociales se manejaron desde una lógica partidista y clientelar a través de los Consejos de Poder Ciudadano (СPC). ${ }^{22}$ Por ello muchos analistas mostraron su preocupación en cuanto a los mecanismos de focalización empleados en estos programas y su temor de que el gobierno usara los beneficios sociales para construir una base partidaria cautiva en vez de abordar conscientemente la lucha contra la pobreza y fortalecer la autonomía ciudadana.

Por otro lado, fuentes afines al gobierno expusieron que, gracias a las políticas de promoción social implementadas en 2008, 44.000 familias que vivían en zonas rurales se vieron beneficiadas con agua potable y electrificación, a la vez que se construyeron 10.426 viviendas, 234 en comunidades rurales. Asimismo, el programa Usura Cero dio crédito a 16.000 socias; las campañas de alfabetización redujeron el analfabetismo del $22 \%$ en 2006 al $13 \%$ en diciembre de 2008. En cuanto a infraestructuras, se expuso que se había rehabilitado, ampliado y mantenido $9.000 \mathrm{~km}$ de carreteras y once puentes en la Región Autónoma del Atlántico Norte, que es la más abandonada del país. Respecto al sector productivo el programa Hambre Cero entregó el Bono Productivo Alimentario a 20.000 familias desde mediados de 2007 hasta diciembre de 2008 y se triplicó el crédito agrario para la pequeña y mediana producción. ${ }^{23}$

Antes de finalizar este epígrafe es importante exponer que desde la llegada de Ortega al poder se dio una preocupante "batalla por los datos" a raíz de la existencia de cifras contradictorias en materia socioeconómica en función de las fuentes. Con todo, sí se ha aceptado que la inflación acumulada (el IPC) durante el año 2008 fue del 13, 8\% y que el precio de la canasta básica pasó de 3.046,10 córdobas en enero del 2007 a 8.636,4 en junio de 2008. Fruto de ello, los datos -también los oficiales- han constatado que a pesar de los esfuerzos realizados la pobreza a nivel nacional ha crecido por primera vez en una década.

\section{La política exterior: ¿Un nuevo protagonismo internacional?}

La política internacional en 2008 siguió la misma lógica del primer año de la administración Ortega, pero en éste se ahondó el distanciamiento entre del gobierno con la comunidad internacional de donantes y con las ONGs.

Respecto a las continuidades es preciso nombrar que, a nivel regional, el gobierno de Ortega mantuvo y profundizó su alianza con el conjunto de países que conforman el

21 El diseño de los presupuestos de 2008 fue muy semejante al de años anteriores, especialmente respecto al pago de la deuda interna y a las negociaciones con el FMI.

22 Aunque el formato de los CPC recuerda a los Comités de Defensa Sandinista (CDS) de la década revolucionaria, si se analizan de forma más pormenorizada (y en su forma de actuar) se puede concluir que tienen muchos puntos en común con las tradicionales redes clientelares en base a las cuales se distribuyen beneficios públicos.

23 Datos obtenidos de "La Gaceta de Noticias" presente en la página: www.presidencia.gob.ni 
ALBA, participando en la VI Cumbre celebrada en Caracas y organizando en Managua, el día 7 de mayo, una Cumbre sobre Seguridad Alimentaria (también en el marco de dicha alianza) en la que participó el Presidente de Bolivia, Evo Morales. Alrededor de esta "alianza estratégica" el gobierno nicaragüense obtuvo notables réditos económicos (tal como ya se ha señalado) pero Daniel Ortega también presenció algunas situaciones incómodas, como la acontecida durante la toma de posesión del nuevo Presidente de Paraguay, Fernando Lugo, que se celebró el día 15 de agosto. En dicha toma de posesión Ortega no pudo llegar a la ciudad de Asunción debido a que la Secretaria de la Mujer del nuevo gobierno de Lugo, Gloria Rubin, amenazó con dimitir si llegaba a la ceremonia de toma de poseción un Jefe de Estado sobre el que pesaban acusaciones de abuso sexual, en referencia al "caso Zoilamérica". Finalmente, el anuncio que despertó más recelos en Nicaragua -vinculado al ALBA- fue la declaración de Ortega, realizada a inicios de 2008 y de la que posteriormente no hubo ninguna información adicional, de que se estaba pensando en la creación de unas Fuerzas Armadas del Alba.

Fruto de esta estrecha relación entre Nicaragua y el Alba se comprende la prolongación de la tensión diplomática entre Managua y Bogotá, escenificada en 2008 a través de dos temas. El primero fue contencioso territorial de Nicaragua sobre el archipiélago de San Andrés, y el segundo fue producto del "asilo" que Ortega ofreció a tres estudiantes mexicanos que estaban en un campamento de las FARC en Ecuador, cuando el ejército colombiano lanzó una ofensiva sobre éste.

Fuera de la región la administración Ortega continuó manteniendo cordiales relaciones con Libia e Irán, e inició nuevas relaciones con Rusia. De ésta destaca la aprobación, por parte del gobierno de Irán, de un crédito de 231 millones de dólares para que una compañía eléctrica iraní construyera un megaproyecto en Nicaragua. Respecto a Rusia, cabe señalar la visita que realizó a Managua el Primer Ministro ruso, Igor Sechin, el 17 de septiembre, en la que firmó diversos convenios de cooperación. Sólo a partir de esta nueva aproximación con el gigante euroasiático es posible comprender las razones por las que Nicaragua fue el segundo país del mundo (después de Rusia) en reconocer, el primero de septiembre, la independencia de Abjasia y Osetia el Sur. En este marco no es casual que la administración Bush (al final de su mandato) destinara de embajador a Managua a un viejo halcón, Robert Callahan, quien había tenido un activo rol de apoyo a los "Contras" durante los años ochenta desde la embajada de Tegucigalpa.

En cuanto a la relación entre el gobierno y la comunidad internacional de donantes, destacó el acoso que sufrieron, por parte del gobierno nicaragüense, los embajadores de estos países (sobre todo los de la Unión Europea) a quienes el gobierno de Ortega acusó de estar vinculados a ONGs que blanqueaban dinero. ${ }^{24}$ Esta acusación fue fruto de diversas actuaciones que habían realizado las agencias oficiales de cooperación y diversas ONGs de dichos países y que no habían sido del agrado de la administración Ortega. La primera era que algunas embajadas de los países miembros de la UE informaron al gobierno que

24 Sobre ello destaca la declaración de persona "non grata" del gobierno nicaragüense a la comprometida embajadora sueca en el año 2008. 
sus países dejarían de brindar ayuda a menos que la administración diera un giro en ciertas políticas, entre ellas, la penalización del aborto sin excepción alguna, cosa que el gobierno de Nicaragua tomó como una violación de su soberanía. La segunda era que durante algún tiempo -sobre todo desde la administración de Alemán (1996-2001)-ONGs como Oxfam UK administraron directamente una parte de la ayuda oficial al desarrollo sin pasar por la administración gubernamental. ${ }^{25} \mathrm{Y}$ la tercera y última era que, según Ortega, estos países al ser críticos con el gobierno se alineaban con la oposición y, por lo tanto, trabajaban para desestabilizar al gobierno (Close y Martí 2009).

Obviamente este enfrentamiento con los "países donantes" sería difícil de entender si no fuera por las nuevas fuentes de ingresos monetarios y materiales que obtiene la administración Ortega de los nuevos "países amigos". Unos recursos cuya gestión no requiere de la rendición de cuentas exigida por los tradicionales cooperantes y cuyo monto no es nada despreciable. Un cálculo aproximado sugiere que los fondos disponibles de tan sólo uno de los varios acuerdos de cooperación con Venezuela "casi iguala la ayuda internacional total de occidente que Nicaragua recibe en un año" (Castillo Argüello 2008: 13-14).

\section{ELECCIONES MUNICIPALES: ¿EL FIN DE LA RENDICIÓN DE CUENTAS VERTICAL?}

Las elecciones municipales el 9 de noviembre de 2008, donde casi cuatro millones de nicaragüenses estaban citados a las urnas, constituyen el episodio político más relevante del año y también el que más incrementó la preocupación a muchos nicaragüenses y a la comunidad internacional. ${ }^{26} \mathrm{El}$ desenlace de dichos comicios hizo incrementar el nivel de polarización política ya existente, debido a que se puso en cuestión la única forma de rendición de cuentas existente -la vertical- y mostró la capacidad que mantiene Ortega de controlar agencias y poderes del Estado, y con especial significación el CSE. ${ }^{27}$

Esto ilustra también el escepticismo por parte de los donantes acerca de la capacidad del gobierno para aplicar la ayuda de manera transparente y según los criterios de los donantes.

26 La trascendencia de las elecciones municipales radica en su significado político, por un lado, debido que al tratarse de elecciones intermedias ejercen de "barómetro" del desempeño del gobierno en curso y, por otro, como mecanismo de reclutamiento y retribución de miembros partidarios en el territorio. Sin embargo, en cuanto a las competencias y los recursos de los municipios son limitados, ya que Nicaragua es un país muy centralizado. El gasto que realizan los municipios es un $7 \%$ del gasto total de la República.

27 Desde El Pacto entre Ortega y Alemán en el año 2000 las instituciones que deberían fiscalizar al Poder Ejecutivo se politizaron y, por tanto, puede afirmarse que no existe rendición de cuentas horizontal entre las instituciones del Estado. La otra forma de rendición de cuentas (realizada por los electores y que se ha convenido en llamar "vertical") se erosionó con la "cooptación" y politización (a favor del FSLN) del Consejo Supremo Electoral. Las razones del control de Ortega sobre la autoridad electoral deriva de que, según diversas fuentes, de los siete magistrados que componen el CSE tres mantienen lealtad al FSLN, tres al PLC y uno, el Presidente, es uno de los operadores políticos de "El Pacto" y, por lo tanto, protege los intereses de Ortega y Alemán. A la vez, de las nueve direcciones centrales que tiene la institución cinco "son" sandinistas, tres las controla directamente el Presidente del CSE y una es del PLC. Finalmente, de las 17 delegaciones departamentales, doce están dirigidas por cuadros del FSLN y cinco por cuadros liberales. Además, según la Ley electoral, las 11.808 Juntas Receptoras de Votos están compuestas -entre otros- por miembros de las dos formaciones que quedaron en el primer y segundo puesto en las últimas elecciones (en este caso, las de 2006); y en el caso de las elecciones municipales de 2008 estos miembros pertenecían al FSLN y a la ALN, formación que en 
Durante los meses anteriores al proceso electoral el CSE tomó algunas decisiones que muchos observadores calificaron de preocupantes. Entre éstas destacaron tres de tipo administrativo y tres de hondo calado político. Entre las decisiones administrativas destacaron el aplazamiento de las elecciones en algunas localidades de la Costa Atlántica debido a los destrozos causados por el huracán Félix, que arrasó la zona meses antes; el adelanto de dos meses para cumplir algunos requisitos para la inscripción de candidaturas (avanzando al día 3 de marzo la fecha para presentar alianzas de partidos y al 9 del mismo mes para presentar a los candidatos), y la extensión de la legalidad de cédulas vencidas, hecho que abrió el debate sobre el registro de ciudadanos y la validez del padrón electoral. Y en cuanto a las decisiones de carácter político es preciso señalar, en primer lugar, la cancelación de la personería jurídica del Partido Conservador y del Movimiento Renovador Sandinista por parte del CSE; ${ }^{28}$ en segundo lugar, la resolución en contra de Eduardo Montealegre en el conflicto interno para el control del partido ALN (formación con la que Eduardo Montealegre se había presentado en las elecciones de 2006), dando la razón a un sector próximo al mismo $\mathrm{FSLN}^{29}$ y, finalmente, la restrictiva política de acreditación de comisiones de observadores internacionales. ${ }^{30}$

En este contexto, antes de que empezara la campaña electoral ya hubo importantes manifestaciones en contra de la forma en que el gobierno gestionaba el proceso, destacando una marcha de 20.000 personas en Managua, organizada por una plataforma llamada Coordinadora Civil. Posteriormente, la campaña electoral fue tensa y repleta de incidentes. Hubo sucesos violentos en León días antes de iniciar la campaña, y en Managua durante ésta, además de una intensa movilización de cuadros sandinistas y antisandinistas en casi todo el país. Con esta dinámica la campaña terminó convirtiéndose en una especie de "plebiscito" de los dos años de gestión de Ortega ${ }^{31}$ y el epicentro de la batalla fue la lucha por la alcaldía de Managua, donde Eduardo Montealegre -que se presentó bajo las siglas del PLC_ ${ }^{32}$ creó un movimiento llamado "Vamos con Eduardo" (MCE). A esta plataforma, a la vez, se sumaron adhesiones de otras sensibilidades, consiguiendo el apoyo del Movimiento Renovador Sandinista (MRS) y de los conservadores. Ello fue así

noviembre del 2008 no obedecía a Eduardo Montealegre, sino a personas con vínculos con el FSLN. Sobre este tema véase: http://www.envio.org.ni/articulo/3890.

28 Hecho que generó protestas de la comunidad internacional y múltiples fenómenos de protesta, entre los que destacó la huelga de hambre que realizó la dirigenta del MRS (y ex comandante guerrillera de la Revolución), Dora María Téllez, durante 13 días y que tuvo una importante resonancia internacional.

29 Con ello Montealegre, que se presentó como candidato a la alcaldía de Managua, se quedó sin referente partidario y tuvo que "pactar" con la formación que anteriormente había abandonado (el PLC, controlado por Arnoldo Alemán) para que le admitieran nuevamente y lo postularan como candidato a alcalde de la capital.

30 Muchas comisiones de observadores no tuvieron permiso, entre ellas, se denegó la presencia del Centro Carter, que jugó un rol muy relevante en 1990. Sí se dio que estuvieron presentes, sin embargo, miembros del Protocolo de Tikal y del Protocolo de Quito de observación internacional, de la OEA y del Consejo de Expertos Electorales de América Latina (CEELA).

31 Un plebiscito en un momento en el que -según la Revista Envío- la encuestadora CID-Gallup exponía que sólo el 18\% de los nicaragüenses apoyaban su gestión a mediados del año 2008.

32 Concurrió junto con Enrique Quiñónez (un cuadro liberal tradicional) que figuró como candidato a vicealcalde. 
hasta el punto que se creó una especie de espacio político alrededor de Montealegre, que se conoció como el TCO: "Todos Contra Ortega".

La candidatura de Montealegre, sin embargo, tuvo que sortear algunos problemas para ser efectiva, ya que la fiscalía anticorrupción (controlada por el FSLN) mantuvo hasta el último día la amenaza de que éste, junto con 39 funcionarios más de administraciones anteriores, podía ser aforado ante la justicia por un caso de quiebras bancarias que ocurrieron en Nicaragua durante los años en que Montealegre fue Ministro de los gobiernos Alemán y Bolaños. Pero el gran reto de la oposición era competir en un contexto en el que los recursos públicos fluían con generosidad para los candidatos sandinistas. En el caso de Managua los candidatos del FSLN fueron -a pesar de la opinión de algunos cuadros sandinistas, pero apoyados por la Primera Dama- el boxeador Alexis Argüello y la periodista Daisy Torres, si bien la campaña se centró rápidamente en la misma figura del Presidente Ortega.

Hubo pocas encuestas preelectorales, sin embargo, una de las más fiables, elaborada por la Universidad Centroamericana, otorgaba al PLC una victoria cómoda (de más del 50\%) en Managua y la posibilidad de hacerse con las alcaldías de ocho cabeceras departamentales del país, y daba al FSLN mayor apoyo electoral en las nueve cabeceras restantes. A nivel agregado la encuesta daba un resultado muy ajustado. Frente a los datos que arrojaba dicha encuesta la postura oficial del FSLN, verbalizada por la Primera Dama, fue descalificar el trabajo y decir que la publicación de encuestas era una estrategia opositora para deslegitimar la victoria del FSLN.

En dicha tesitura el reto del FSLN era superar los excelentes resultados que la formación obtuvo en el ámbito local en 2004, en los que concurrió junto con una agrupación de personalidades independientes que se llamaba Convergencia Nacional. En las elecciones municipales de 2004 el FSLN se hizo con 87 alcaldías -de los 153 municipios existentes-, y entre ellas las 14 de las 17 capitales departamentales.

En las de noviembre de 2008, según los datos oficiales (sobre los que hay una gran discusión), ${ }^{33}$ el FSLN obtuvo 105 alcaldías de los 150 en juego, ${ }^{34}$ y entre ellas las de Managua y León, y todas las cabeceras departamentales con la excepción de Granada, Boaco, Bluefields. ${ }^{35}$ Por otro lado, la oposición se alzó con la victoria en 37 municipios. Pero el proceso fue puesto en cuestión por la oposición. Los resultados en Managua y León fueron los más contestados, esgrimiendo que las actas de sus apoderados daban una clara victoria a Montealegre a pesar de que ya a las 11 de la noche del domingo 9 las calles se llenaron de simpatizantes sandinistas con el fin de celebrar la "victoria".

33 Véase, por ejemplo, la página web www.voto2008.org - elaborada por los seguidores de Eduardo Montealegreen la que exponen los resultados por distrito que ellos creen verdaderos de la elección de Managua. Cabe señalar que en siete municipios de la Costa Atlántica no se celebraron elecciones.

35 Hasta la fecha no se han hecho estudios sobre el comportamiento electoral de los nicaragüenses en las elecciones municipales debido a que existieron pocas encuesta y también porque las denuncias de irregularidades empeñaron su credibilidad. A la vez, también es preciso anotar que este tipo de elecciones se considera de "segundo orden" y por ello se les dedica menor atención que a las presidenciales o legislativas. 
Resultados electorales en los municipios más importantes del país

\begin{tabular}{lrrrrr}
\hline \multicolumn{1}{c}{ Municipio } & \multicolumn{1}{c}{ PLC } & FSLN & ALN & AC & PRN \\
\hline Managua & 185.343 & 231.679 & 3.002 & 2.036 & 2.004 \\
León & 29.921 & 34.355 & 225 & 1.690 & 236 \\
Masaya & 27.452 & 27.743 & 1.599 & 184 & 490 \\
Matagalpa & 22.502 & 28.018 & 890 & 88 & 221 \\
Estelí & 14.224 & 26.826 & 735 & 71 & 169 \\
Chinandega & 15.390 & 20.393 & 2291 & 144 & 154 \\
Granada & 21.798 & 15.043 & 1.863 & 391 & 341 \\
Tipitapa & 17.927 & 19.649 & 586 & 81 & 654 \\
Jinotega & 17.176 & 18.123 & 326 & 253 & 264 \\
Nindirí & 14.022 & 14.095 & 3.070 & 12 & 33 \\
Boaco & 11.449 & 9.731 & 192 & 31 & 95 \\
Ciudad Sandino & 9.421 & 15.249 & 2.567 & 208 & 187 \\
Siuna & 14.174 & 9.876 & 152 & 0 & 107 \\
Nueva Guinea & 12.518 & 6.284 & 908 & 53 & 516 \\
Bluefields & 8.376 & 7.113 & 407 & 30 & 473 \\
Rama & 9.795 & 6.210 & 407 & 30 & 473 \\
Juigalpa & 7.132 & 8.466 & 165 & 33 & 323 \\
Chichigalpa & 7.175 & 9.905 & 793 & 23 & 60 \\
Puerto Cabezas & 6.172 & 8.030 & 683 & Yatama 6.694 & 77 \\
\hline
\end{tabular}

(*) Yatama es una formación política propia de la Costa Caribe de Nicaragua que representa a los pueblos indígenas allí radicados (mayoritariamente miskitos, pero también sumos, ramas y creoles).

Fuente: CSE, publicado el 22 de enero de 2009. Están marcados en fondo gris las cabeceras departamentales donde la oposición ganó.

En los días siguientes a las elecciones hubo agrios enfrentamientos en las calles entre sandinista y antisandinistas. La oposición llamó a la desobediencia civil y los simpatizantes del FSLN ocuparon las vías públicas con el objeto de disuadir cualquier manifestación. El acontecimiento más violento se dio el día 16 de noviembre en una marcha de protesta en León, donde hubo enfrentamientos que se saldaron con decenas de heridos.

En este marco cabe destacar las declaraciones del Secretario General de la OEA, José Miguel Insulza, realizadas el día 11 de noviembre de 2008, en las que expresó su preocupación por la violencia callejera y las denuncias de la oposición. También los obispos nicaragüenses se manifestaron en la misma dirección, a la par que el Centro Carter solicitó al CSE una revisión de los resultados con las debidas garantías. Sin embargo, el CSE no tuvo en cuenta las recomendaciones y el día 13 de noviembre publicó los resultados oficiales. Las irregularidades y la tensión que generó el proceso fueron tales que se discutió sobre los comicios nicaragüenses el 20 de noviembre en la sede de la OEA, aunque tanto la moción de denuncia (norteamericana) como la de apoyo (del gobierno de Nicaragua) fueron retiradas por falta de apoyo. 
Los comunicados de las comisiones de observadores fueron contradictorios. Por un lado, los observadores electorales del CEELA (Consejo de Expertos Electorales de Latinoamérica) y del Protocolo de Tikal (integrado por magistrados electorales de Centroamérica y del Caribe) informaron que durante el proceso de votación no se produjeron anomalías significativas que pudieran alterar el resultado de las votaciones. Y, por otro, la organización nicaragüense Ética y Transparencia, que auspiciaba una comisión de observadores nacionales, declaró que se trataba de las elecciones "menos trasparentes y más conflictivas de la historia", a la par que declaraba que había una gran cantidad de anomalías e irregularidades en el $32 \%$ de las Juntas Receptoras de Votos de todo el país, y que éstas se caracterizaban por su gravedad y por afectar siempre de forma negativa a la oposición. ${ }^{36}$

De lo expuesto es posible afirmar que el desenlace del proceso supuso un duro golpe a la credibilidad de la institución electoral y a la capacidad de hacer efectiva la rendición de cuentas vertical (que compete a la decisión de los ciudadanos en las urnas), fenómenos que fueron especialmente graves debido a que, desde la existencia de El Pacto, la rendición de cuentas horizontal se ha visto notablemente mermada por la politización de todas las instituciones estatales.

\section{EVALUACIÓN SOBRE EL FUNCIONAMIENTO DE LA DEMOCRACIA}

No es fácil intentar esbozar una evaluación de la democracia en Nicaragua en pocas líneas, a la par que dicho ejercicio supone un trabajo que trasciende al análisis de lo acontecido en un año. Sin embargo, partiendo del esquema que proponen Levine y Molina (2006) y tomando el año 2008 como referencia, intentaremos interpretar cuál es "momento" de la democracia nicaragüense centrándonos en sus debilidades, en el nivel de participación política de los ciudadanos, en la capacidad de respuesta de los gobiernos a las demandas y, finalmente, respecto a soberanía del Estado.

En primer lugar es posible afirmar que uno de los mayores problemas de la democracia nicaragüense ha sido la continua modificación de las reglas de juego (sobre todo de las dos más relevantes, la Constitución y la Ley electoral) según los intereses de los caudillos políticos. Además, desde El Pacto firmado en el año 2000 se ha incrementado un fenómeno que merma considerablemente la calidad de un sistema democrático, a saber, la politización de la administración del Estado, del Poder Judicial y de todas sus agencias "autónomas" estatales. A resultas de ello durante la última década ha sido prácticamente imposible hablar de una rendición de cuentas horizontal. Pero además de los dos problemas señalados -la maleabilidad de las reglas y la politización de las instituciones- cabría añadir un tercero detectado el año 2008: la actuación partidista del Consejo Supremo Electoral en unos comicios, limitando así la rendición de cuentas vertical.

36 El informe está disponible en el enlace (consulta del día 12 de marzo de 2009):: http:/ / www.enlaceacademico. org/uploads/media/informe_final_electoral_2008.pdf 
Respecto a la participación es preciso señalar que la abstención en las elecciones Presidenciales ha sido una de las más bajas de América Latina. ${ }^{37}$ Pero al hablar de participación política es importante también apuntar la importancia que ha tenido en el país, además del voto, la movilización social en las calles. Después de la década de los ochenta, en que el régimen revolucionario impulsó grandes movilizaciones, las protestas callejeras y la toma de espacios públicos han devenido herramientas comunes del repertorio de acción colectiva de los nicaragüenses. En este sentido es importante tener en cuenta la capacidad de confrontación de diversos sectores de la sociedad. Y no sólo de los colectivos populares organizados en centrales sindicales o de los movimientos comunitarios, sino también amplios sectores de la clase media organizada en redes de ONGs -tal como ocurre con el movimiento feminista y con los movimientos ciudadanos que se han manifestado durante la última década, primero contra El Pacto durante la administración Alemán y actualmente contra algunas medidas de la administración Ortega.

En cuanto a la capacidad de respuesta de los gobiernos a las demandas ciudadanas es preciso señalar la débil capacidad de la administración del Estado, tanto en lo que se refiere a sus recursos y, a la gestión de los mismos, como a la posibilidad de insertarse (y negociar) en un mundo global. Además, desde 1990 hasta 2006 las diversas administraciones liberales redujeron más el rol del Estado y dieron prioridad al mercado, abriendo la economía al exterior y reduciendo drásticamente la prestación de políticas sociales. ${ }^{38}$ Los datos de desarrollo socioeconómico y humano de las últimas décadas muestran la poca capacidad para crear riqueza y la aún menor de luchar contra la pobreza. Desde la llegada de Ortega se expuso la voluntad de fortalecer y dar un nuevo rol para el Estado, pero hasta la fecha los cambios experimentados se han limitado a la implementación de políticas sociales focalizadas que han tenido un impacto muy limitado y que, además, ha reforzado el clientelismo.

Finalmente, en lo que hace al elemento de la soberanía, Nicaragua presenta rasgos contradictorios. Por un lado, existe un pleno control del gobierno de los cuerpos de seguridad y una notable capacidad de tener presencia a lo largo del territorio (con algunas lagunas en la Costa Caribe), ${ }^{39}$ a la vez que tiene la capacidad de mantener unos índices bajos de criminalidad y violencia, al contrario de lo que ocurre en países vecinos como El Salvador, Honduras y Guatemala. Por otro lado, cabe señalar la importante injerencia de gobiernos extranjeros en la vida política del país: la permanente gravedad del departamento de Estados Unidos y la presencia de los intereses venezolanos durante los últimos años no son más que un nuevo capítulo de esta dinámica. En esta misma dirección cabe añadir

37 La polarización en las elecciones de primer grado ha supuesto que la abstención fuera limitada: entre el 13\% y el 33\%, si bien esta última -que se registró en 2006- estaba sobredimensionada debido a que el censo no estaba depurado y a que los inmigrantes a Costa Rica y a los Estados Unidos no pudieron votar. Otra cuestión es la participación en las elecciones municipales o regionales, donde la participación oscila entre el 50 y el $60 \%$ del censo.

38 Desde el año 1990 el Estado nicaragüense abandonó la "matriz sociopolítica" llamada "Estado-céntrica" por otra que favorecía las relaciones mercantiles. En cierta forma la calificación de Neoliberal Market-Driven and Multicentered acuñada por Garretón (ed., 2003) encaja perfectamente al rol ejercido por el Estado nicaragüense durante las dos últimas décadas.

39 Véase, en este sentido, el excelente libro de Frühling, González y Peter-Buvollen (2007). 
la importancia que tiene la cooperación internacional para el combate contra la pobreza y para el desarrollo del país, mostrando la gran vulnerabilidad que tiene Nicaragua.

De todo ello es posible concluir que la democracia en Nicaragua tiene algún activo si se analiza en una perspectiva histórica. Con todo, abundan los pasivos, algunos de ellos de carácter estructural -como es la debilidad del Estado y su administración en el entorno competitivo de la economía global- y otros de carácter coyuntural, si bien estos últimos pueden ser atribuidos a la cultura política y pueden terminar permaneciendo en el sistema política en detrimento de la calidad de la democracia. Entre éstos destacan la polarización política, la politización de las instituciones (que ha mermado gradualmente la capacidad de rendir cuentas del Estado y de sus representantes) y la dinámica partitocrática de dos formaciones (el FSLN y el PLC) que han terminado monopolizando la vida política y que están controladas por dos líderes (Daniel Ortega y Arnoldo Alemán) que actúan de forma patrimonial. De todo lo expuesto podemos concluir que lo acontecido durante el año 2008 en Nicaragua no ha supuesto ningún avance en el proceloso camino de mejorar la calidad democrática del país.

\section{REFERENCIAS}

Castillo Argüello, Ricardo. 2008. "La economía política de los petrodólares". Confidencial, 606, 19 al 25 de octubre, 14.

Close, David. 2004. "President Bolaños Runs a Reverse, or How Arnoldo Alemán Wound Up in Prison”. En Close, David y Deonandan, Kalowatie eds. 2004. Undoing Democracy. The Politics of Electoral Caudillismo. NY: Lexington Books.

Close, David y Salvador Martí. 2009, en prensa. Nicaragua y el FSLN: Un análisis de la realidad política desde 1979. Edicions Bellaterra: Barcelona.

Kampwirth, Karen. 2004. "Alemán's Wa ron the ONG Community". En Close, David y Deonandan, Kalowatie eds. Undoing Democracy. The Politics of Electoral Caudillismo. NY: Lexington Books.

Deonandan, Kalowatie. 2009, en prensa. "Feminismos, antifeminismo y la lucha del aborto terapéutico. La memoria y las consecuencias inesperadas de la revolución". En David Close y Salvador Martí i Puig. Nicaragua y el FSLN: Un análisis de la realidad politica, 1979-2006. Barcelona: Edicions Bellaterra.

Frühling, Pierre, Miguel González y Hans Peter-Buvollen. 2007. Etnicidad y nación. El desarrollo de la autonomía de la Costa Atlántica de Nicaragua. 1987-2007. Guatemala: F\&G Editores.

Garretón, Antonio Ed. 2003. Latin America in the Twenty-First Century: Toward a New Sociopolitical Matrix. Miami: North/South Center Press.

Levine, Daniel H. y José Enrique Molina. 2006. “La calidad de la democracia en América Latina: una visión comparada". Paper presentado en el Congreso de la Latin American Studies Association, LASA, Montreal.

Martí i Puig, Salvador. 1997. Nicaragua 1977-1996. La revolución enredada. Madrid: Libros de la Catarata. .2008a. "El regreso del FSLN al poder: ¿Es posible hablar de realineamiento electoral en Nicaragua?". Revista Política y Gobierno, XIV (1).

2008b. "El regreso de Ortega: los primeros pasos de su segunda administración". Revista de Ciencia Política 28(1): 287-303.

Spalding, Rose. 2009, en prensa. "Las políticas contra la pobreza". En Close, David y Salvador Martí eds. Nicaragua y el FSLN: Un análisis de la realidad política desde 1979. Edicions Bellaterra: Barcelona.

Stahler-Sholk, Richard. 1994. "El ajuste neoliberal y sus opciones: la respuesta del movimiento sindical nicaragüense". Revista Mexicana de Sociología 3:59-88. 


\section{RECURSOS ELECTRÓNICOS UTILIZADOS}

www.envio.org.ni

www.elnuevodiario.org,ni

www.laprensa.org.ni

www.confidencial.org.ni

www.presidencia.gob.ni

www.asamblea.gob.ni

http:/ / www.lavozdelsandinismo.com/

Salvador Martí es Profesor titular de Ciencia Política de la Universidad de Salamanca y miembro del Institut Barcelona d'Estudis Internacionals (IBEI).

[E-mail: smarti@usal.es] 
